\title{
Effect of Ultraviolet Radiation on Conidia Survival of Potential Mycotoxigenic Aspergillus Species
}

\author{
Esther Garcia-Cela (I), Antonio J Ramos (I), Vicente Sanchis (I), \\ Sonia Marin (I) \\ (I) UdL - University of Lleida (Rovira Roure 191; 25198 Lleida ESPANHA), (II) UdL - University \\ of Lleida-Agrotecnio (Rovira Roure 191;25198 Lleida (Spain))
}

\section{Resumo}

Bio-geographical differences in fungal profile infection have been observed in the world, proving that the climatic conditions are decisive in the colonization. Nevertheless environmental conditions regarding to fungal development have been generally described in terms of temperature (T) and relative humidity $(\mathrm{RH})$, while other important factors as ultraviolet radiation (UV) have received lower attention, despite the International Agencies consider that variations in UV-B radiation can have large effects on plant interactions with pests, with important implications for Food Security and Food Quality. To assess the effects of UV-A and UV-B radiation on survival of potentially mycotoxigenic fungi under in vitro simulated field conditions, conidial suspensions of isolates of A. carbonarius, A. tubingensis, A. flavus, A. parasiticus, A. westerdijkiae and A. ochraceus were filtered under vacuum onto individual sterile cellulose acetate filter membranes (pore size 0.45 \&micram) and dried at $37{ }^{\circ} \mathrm{C}$ overnight. Then spores were incubated under $\mathrm{T}$ and light cycles of $8 \mathrm{~h}$ darkness $\left(20^{\circ} \mathrm{C}\right)$ and $16 \mathrm{~h}$ of light $\left(5 \mathrm{~h}\left(\right.\right.$ from 20 to $\left.30^{\circ} \mathrm{C}\right)+6 \mathrm{~h}\left(30^{\circ} \mathrm{C}\right)+5 \mathrm{~h}$ (from 30 to $20^{\circ} \mathrm{C}$ ) for 15 days. Additionally, six hours of exposure per day to UV-A or UV-B radiation were included in the light exposure. UV doses used were $1.7 \pm 0.2 \mathrm{~mW} / \mathrm{cm} 2$ of UV-A (peak, $365 \mathrm{~nm}$ ) and $0.10 \pm 0.2$ $\mathrm{mW} / \mathrm{cm} 2$ of UV-B (peak, $312 \mathrm{~nm}$ ) slightly higher than present sunlight UV mean values. Each five days (5th, 10th and 15th day) conidia were dislodged from two control and two irradiated membranes into $100 \mathrm{~mL}$ 
sterile peptone $(0.1 \% \mathrm{w} / \mathrm{v})$ solution using a stomacher. Subsequently, a seriate dilution was carried out and $100 \mu \mathrm{L}$ of each dilution were plated onto DRBC and incubated at $25^{\circ} \mathrm{C}$ in darkness for two days. Afterwards the colonies were counted. The intrinsic decrease of viability of conidia along time was accentuated when they were UV irradiated. UV-B radiation was more harmful than UV-A. Conidial resistance to UV light was more marked in Aspergillus section Nigri (black), followed by Flavi (green) and Circumdati (yellow) and hence the pigmentation of the conidia could be related to UV sensitivity. Interestingly, different resistance was observed between species belonging to sections Flavi and Nigri. As conclusion, a possible increase in UV radiation related with climate change could lead to a reduction in the inoculum present in the field and even it could exert a selective selection on fungal species.

Palavras-Chave: Aspergillus, Ultraviolet radiation, Climate change Agência de Fomento: 